\title{
DIFFERENT CLINICAL AND ANGIOGRAPHIC FINDING IN A SET OF IRANIAN IDENTICAL TWIN: A CASE REPORT
}

\author{
${ }^{1}$ Mohammad Shojaie, ${ }^{2}$ Mohammad Yasin Karami, \\ ${ }^{3}$ Mahdi Karimyar Jahromi, ${ }^{1}$ Abdolreza Sotoodeh Jahromi and ${ }^{2}$ Alireza Makarem \\ ${ }^{1}$ Department of Internal Medicine, Research Center for Cardiovascular Atherosclerosis, \\ ${ }^{2}$ Department of Student Research Committee, \\ ${ }^{3}$ Department of Nursing, \\ Jahrom University of Medical Sciences, Jahrom, Iran
}

Received 2013-08-17, Revised 2013-08-29; Accepted 2013-09-16

\begin{abstract}
Both genetic and environmental causes are involved in developing of Coronary Artery Disease (CAD), but there is a notable controversy about the environmental and genetic factors that play a role in the pathogenesis of CAD. The value of each individual risk factor on CAD and the incidence of coronary artery disease in identical twins are indefinite. To find out genetic mechanisms of coronary heart disease, the assessment of twins study may help us. On the other hand the natural history of coronary artery disease in an asymptomatic twin with a symptomatic counterpart is undistinguishable. We present a case report about different coronary angiographic findings on an Iranian identical Lady Twin with different clinical outcome in access to incidence of CAD in twins. Based on our patient and the information on other patients, the asymptomatic twins of symptomatic counterparts need aggressive assessment and management for occult coronary artery disease.
\end{abstract}

Keywords: Identical Twin, Coronary Artery Disease, Coronary Angiography

\section{INTRODUCTION}

Atherosclerosis is the combined result of environmental factors such as infection (Pourahmad et al., 2009) and personal predispositions (Jahromi et al., 2010). Factors such as low serum adiponectin (Shojaie et al., 2009a) and annexin V levels (Shojaie et al., $2009 \mathrm{~b}$ ) are a part of involving factors in atherosclerosis. The genetics of arteriosclerosis and Coronary Artery Disease (CAD) have been an important research point for several years (Hara et al., 2013). The degree to which, every genetic and environmental factors contributes to the development of CAD is not definitely known (Nilsson, 2012). The incidence of coronary artery disease in identical twins is unclear. Although, researching on monozygot twin with $\mathrm{CAD}$ is helpful for understanding the role of genetics in atherosclerosis. Only a few case reports have showed angiographic findings of monozygotic twin pairs with coronary heart disease (Qi et al., 2013). We report a monozygot twin with different clinical and angiographic finding.

\subsection{Case Presentation}

Twin A: Twin A is a 51 years old obese woman; she was presented with typical chest pain at sleep 3 years ago. She had been admitting in Motaharee Hospital affiliated to Jahrom University of Medical Sciences with diagnosis of Extensive anterior Myocardial Infarction (MI). She has some coronary risk factors such as hypercholesterolemia, obesity (Body Mass Index: $31 \mathrm{~kg} \mathrm{~m}^{-2}$ ), hypertension (BP: 140/80) and diabetes mellitus. Coronary angiographic studies showed three vessels disease including significant narrowing of the Left Ascending Artery (LAD) in the proximal segment and after first diagonal and severe Corresponding Author: Mohammad Yasin Karami, Department of Student Research Committee, Jahrom University of Medical Sciences, Jahrom, Iran 
stenosis in the first and second diagonal (D1, D2) and Obtus Marginals arteries (OM) and other stenosis were seen in the mid part of right coronary artery (Fig. 1). Twin A had undergone Coronary Artery Bypass Graft (CABG) including Left Internal Mammary Artery (LIMA) to LAD and two saphenous vein grafts to RCA and marginal branch of the circumflex artery. Her postoperative period was uneventful and she was discharged from hospital at the sixth day after surgery. The patient has a good condition now.

Twin B: Twin B is a 51 year-old woman, who was presented with chest pain 2 week after news of CABG in her sister and admitted with the impression of unstable angina. She had some coronary risk factors such as hypercholesterolemia, obesity (Body Mass Index-BMI: $31.2 \mathrm{~kg} \mathrm{~m}^{-2}$ ) and hypertension (BP: 150/80). Angina pain progressed in hospital course and due to this she was referred for coronary angiography. Coronary angiography showed only significant stenosis in Left Anterior Descending artery (LAD) (Fig. 2). Twin B had undergone coronary angiography and after 6 months Percutaneous Coronary Intervention (PCI) and stenting was done for her. The patient has a good condition now.

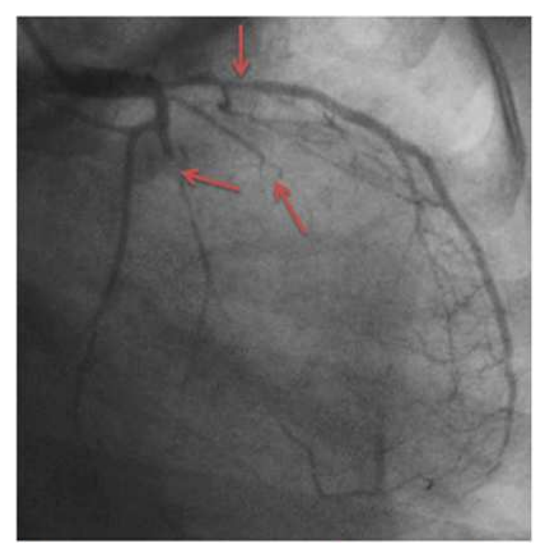

Narrowing in proximal segment of the LDA and severe stenosis in the D1, D2, OM

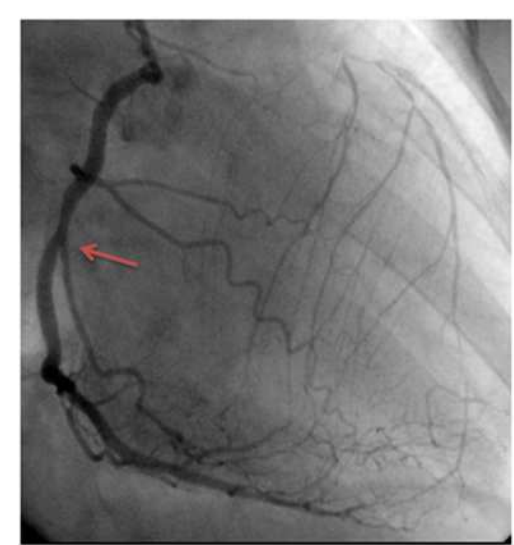

Stenosis in the middle segment of RCA

Fig. 1. Coronary angiography of Twin A

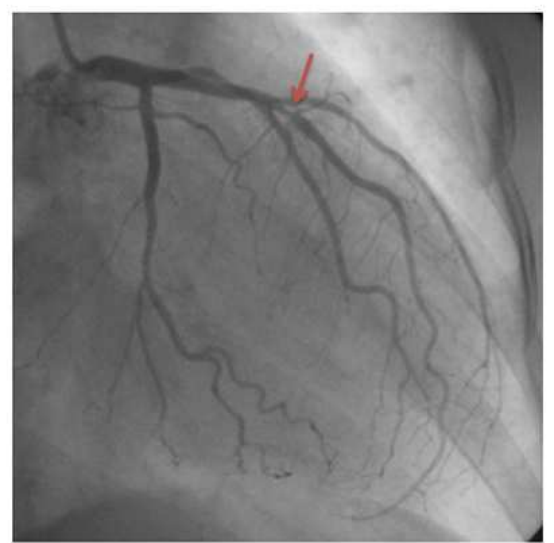

Severe narrowing in the middle segment of LAD

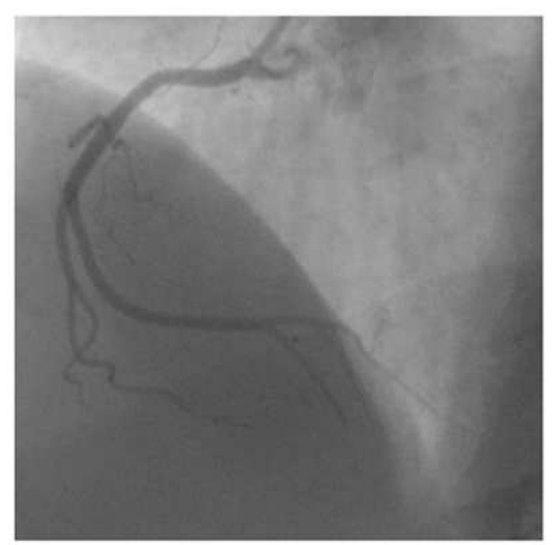

Fig. 2. Coronary angiography of Twin B 


\section{DISCUSSION}

In our report, a pair of twin developed CAD at the same age with difference in coronary risk factors, coronary event and also in angiographic findings. One of our twins had three vessels disease that undergone CABG and another was only single vessel disease that PCI was done for her (Table 1).

Frings et al. (2000) Study on the angiographic coronary anatomy in three monozygotic and three dizygotic twin pairs did not have any evidence in support of the hypothesis in which the coronary blood supply is strictly determined by heredity factors. They compared the location of the stenosis in monozygotic twin pairs with those in dizygotic pairs and cannot confirm a strict heredity of the location of coronary lesions. They concluded that the dominance pattern of the coronary blood supply or the locations of coronary lesions are not identical in "identical twins" with coronary artery disease.

In opposite of this, Gullu et al. (2007) strongly suggested that genetic is highly related not only on the development of coronary artery anatomy but also on occurrence of atherosclerotic lesions in closed similar regions. Kanda et al. (1991) presented a pair of twin with painless MI and suggested possible effect of genetic factors in atherogensis. A side-by-side comparison of twin-pair angiograms reveals the close similarity on location and character of lesions observed in twins from this report (Qi et al., 2013).

Some studies suggested that development of coronary artery branches might be independent from genetics effects (Nilsson, 2012).

Gullu et al. (2007) strongly believed that genetic is highly related not only on the development of coronary artery anatomy but also on occurrence of atherosclerotic lesions in the same regions. Our patients had different risk factors same as the Gullu's report but also opposite, with different angiographic results. We believed that with few twin cases and complex manner of atherosclerosis, it is difficult to discuss about it. Therefore, the same reports with larger numbers of twins are helpful to investigate genetics influence of atherosclerosis.

Hara et al. (2013) study showed that genetic factors have influence in the development of coronary anatomy or expression of coronary disease. However, age at first coronary event, coronary event and coronary risk profile are highly concordant. Marenberg et al. (1994a) suggest that in twins with younger ages, the death from coronary heart disease is influenced by genetic factors in both men and women. The results also imply that the genetic effect decreases at older ages.

Benedict (1958) described a set of twins with CAD. His report mentioned several points of interest including young age, similar electrocardiogram changes and development of coronary events with a small difference in time (6 months). It was considered that a high index of suspicion on occult CAD should be maintained in the asymptomatic twins if a coronary event has occurred in the other twin (Marenberg et al., 1994b). Similarly in our report the time of presentation of twin B was just about six month after twin A. Five publications included of 18 monozygotic twin pairs with angiographic assessment of CAD were presented from 1975 to 2009 (Gullu et al., 2007; Douglas et al., 2009). Among these, most patients were men (13 of 18). Most of twins had the same coronary risk factor (10 pairs) and similar coronary artery involvement (13 pairs). But only 7 pairs had the same risk factor associated with similar coronary artery involvement. Also 2 pairs had different risk factor with different coronary artery involvement similar to our report. Discordance between coronary artery involvements in twins in our study may be due to atherosclerotic lesions is independent from genetic as some mention studies suggested.

Table 1. Clinical and angiographic characteristics of identical twin with coronary artery disease

\begin{tabular}{lll}
\hline Twin & A & B \\
\hline Sex & F & F \\
Coronary Risk factors & Obesity, DM, HLP, HTN & Obesity, HLP, HTN \\
BMI $\left(\mathrm{kg} / \mathrm{m}^{2}\right)$ & 31 & 31.2 \\
Age of Coronary Event (yrs) & 48 & 48 \\
Coronary event & MI & AP \\
Coronary Angiography & $3 \mathrm{VD}$ & $1 \mathrm{VD}$ \\
Procedure & CABG & PCI \\
\hline
\end{tabular}

$(\mathrm{AP}=$ Angina Pectoris; $\mathrm{BMI}=$ Body Mass Index; $\mathrm{CABG}=$ Coronary Artery Bypass Graft; DM $=$ Diabetes Mellitus; HLP $=$ Hyperlipidemia; HTN = Hypertension; MI = Myocardial Infarction; 3VD = three Vessel Disease; 1VD = single Vessel Disease; PCI $=$ Percutaneous Coronary Intervention) 
We must be notice that various genes are involved in atherosclerosis and genes study didn't investigate in any twins reports. For that, it is difficult to describe effect of genetic in twins with CAD.

We recommended cardiac evaluation for the asymptomatic twin, especially if the symptomatic pair's lesion is significant as it was the case in our patient.

\section{COCLUSION}

Discordance between coronary artery involvements in twins may be due to atherosclerotic lesions is independent from genetic as some mention studies suggested. Further studies with larger numbers of twin pairs are needed to evaluate influence of genetic and environmental in CAD.

\section{ACKNOWLEDGEMENT}

We thanks the patients and their family for their cooperations in this study.

\section{REFERENCES}

Benedict, R.B., 1958. Coronary heart disease in identical female twins. Am. J. Med., 24: 814-819. DOI: 10.1016/0002-9343(58)90383-8

Douglas, H., C.G. Hanratty and N.A. Herity, 2009. Coronary artery disease: Anatomy and presentation in identical twins. Ulster Med. J., 78: 187-190. PMID: 19907689

Frings, A.M., B. Mayer, W. Bocker, C. Hengstenberg and D. Willemsen et al., 2000. Comparative coronary anatomy in six twin pairs with coronary artery disease. Heart, 83: 47-50. DOI: 10.1136/heart.83.1.47

Gullu, A.U., M. Kizilay, M. Ates and M. Akcar, 2007. The comparison of angiographic lesions and clinical outcomes in identical twins. Interactive CardioVascular Thoracic Surgery, 6: 575-576. DOI: 10.1510/icvts.2007.153445

Hara, M., Y. Sakata and H. Sato, 2013. Genetic factors in myocardial infarction. Rinsho Byori., 61: 176183. PMID: 23672096
Jahromi, A.S., M. Shojaie, S. Dana and A. Madani, 2010. Anti-cardiolipin antibodyin acute myocardial infarction. Am. J. Immunol., 6: 11-14. DOI: 10.3844/ajisp.2010.11.14

Kanda, T., S. Ohshima, K. Yuasa, T. Toyama and S.I. Takase, 1991. Painless myocardial infarction in identical diabetic twins. Japanese J. Med., 30: 170174. DOI: 10.2169/internalmedicine1962.30.170

Marenberg, M.E., N. Risch, L.F. Berkman, B. Floderus and U. De Faire, 1994a. Genetic susceptibility to death from coronary heart disease in a study of twins. New England J. Med., 330: 1041-1046. DOI: 10.1056/NEJM199404143301503

Marenberg, M.E., N. Risch, L.F. Berkman, B. Floderus and U. de Faire Genetic, 1994b. Susceptibility to death from coronary heart disease in a study of twins. N. Engl. J. Med., 330: 1041-1046. DOI: 10.1056/NEJM199404143301503

Nilsson, P.M., 2012. Genetic and environmental determinants of Early Vascular Ageing (EVA). Curr Vasc Pharmacol., 10: 700-701. PMID: 23259558

Pourahmad, M., A.S. Jahromy and M. Shojaei, 2009. Association of mycoplasma pneumoniae infection with myocardial infarction. Am. J. Immunol., 5: 8488. DOI: 10.3844 /ajisp.2009.84.88

Qi, L., Q. Qi, S. Prudente, C. Mendonca and F. Andreozzi et al., 2013. Association between a genetic variant related to glutamic acid metabolism and coronary heart disease in individuals with type 2 diabetes. JAMA, 310: 821-828. DOI: 10.1001/jama.2013.276305. PMID: 23982368

Shojaie, M., A. Sotoodah and G. Shafaie, 2009a. Is adiponectin associated with acute myocardial infarction in Iranian non obese patients. Lipids Health Dis., 8: 17-17. DOI: 10.1186/1476-511X-8-17

Shojaie, M., A. Sotoodah, S. Roozmeh, E. Kholoosi and S. Dana, 2009b. Annexin V and anti-Annexin V antibodies: Two interesting aspects in acute myocardial infarction. Thrombosis J., 7: 13-13. DOI: $10.1186 / 1477-9560-7-13$ 